beobachtet haben, wurde das ESR-Spektrum des Bis-(4-fluor-phenyl)-stickoxids bei verschiedenen Temperaturen aufgenommen (Tab. 2). Es ergab sich eine schwache Temperaturabhängigkeit von $a_{\mathrm{N}}$ und gegenläufig von $a_{\mathrm{F}}$, die zwar den Ergebnissen von Scheidler und Bolton entspricht, aber zu gering ist, um daraus weitergehende Schlüsse abzuleiten.

\begin{tabular}{|c|c|c|}
\hline Temperatur & $a_{\mathrm{N}}$ & $a_{\mathrm{F}}$ \\
{$\left[{ }^{\circ} \mathrm{K}\right]$} & 9,62 & 4,28 \\
293 & 9,60 & 4,28 \\
263 & 9,54 & 4,28 \\
233 & 9,39 & 4,32 \\
203 & $9,45^{*}$ & $--^{*}$ \\
\hline
\end{tabular}

* starke Linienverbreiterung.

Tab. 2. $a_{\mathrm{N}}$ und $a_{\mathrm{F}}$ [Gauß] des Bis-(4-fluor-phenyl)-stickoxids in Toluol: i-Pentan: Methylcyclohexan $=1: 3: 6$.

\section{Experimentelles}

Die ESR-Spektren wurden mit einem Varian-Spektrometer V-4500 (100 kHz-Feldmodulation, 6 Zoll Magnet) aufgenommen. Das Magnetfeld eichten wir direkt mit einem Protonenresonanz-Magnetometer. Zur ESR-Messung wurden die nach Chen, D'Adamo und W $_{\text {ALTER }}{ }^{14}$ dargestellten Diarylamine $(5 \mathrm{mg})$ in Dioxan (5 ml) mit $\mathrm{PbO}_{2}{ }^{15}(10 \mathrm{mg}) 1 \mathrm{Min}$. geschüttelt. Dann wurde zwischen Wasser und $3 \mathrm{ml}$ Xylol aufgetrennt, die Xylollösungen abfiltriert, 10 Min. mit $\mathrm{N}_{2}$ durchspült und i. Vak. abgeschmolzen.

Herrn Prof. Dr. Richard Kuhn danken wir für die Förderung dieser Arbeit.

14 M. M. Chen, A. F. D'Adamo u. R. I. Walter, J. org. Chemistry 26, 2721 [1961].

15 R. Kuhn u. I. Hammer, Chem. Ber. 83, 413 [1950].

Über Ommochrome, XXIII

\title{
Ommidine - ein neuer Typus von Ommochromen aus Orthopteren
}

\section{Bernt Linzen}

\begin{abstract}
Max-Planck-Institut für Biochemie, München (experimenteller Teil der Arbeit), und Zoologisches Institut der Universität München (Ausarbeitung und Niederschrift)
\end{abstract}

(Z. Naturforschg. 21 b, 1038-1047 [1966] ; eingegangen am 27. Mai 1966)

\begin{abstract}
In the compound eyes of the migratory locust, xanthommatin and two other brownish-red screening pigments can be distinguished. The major component has been isolated and named ommidin. This pigment shows both similarities to and marked differences from known ommochromes. By incorporation of labelled precursors it is demonstrated, that it is derived both from tryptophan - and thus that it is an ommochrome - and from methionine. In insects, ommidin seems to be restricted to orthoptera, but it has been identified by paper chromatography in Limulus polyphemus. The red pigment extracted from locust hypodermis has been separated into two components. Although these are extremely labile, it is concluded on the basis of their general behaviour, of their colour in oxidized and reduced states, and of incorporation experiments, that they also belong to the ommochromes. The significance of these findings is discussed.
\end{abstract}

Die Färbung und Zeichnung der Wanderheuschrecken und ihre große Variabilität wird hauptsächlich durch vier Farbstoff-Arten ermöglicht: Carotinoide, Gallenfarbstoffe, Melanine und ein Pigment, das Chauvin ${ }^{1,2}$ als "Acridioxanthin“, GoodwiN ${ }^{3,4}$ als „Insectorubin“ bezeichnet hat. Eine ein-

\footnotetext{
1 R. Chauvin, C. R. hebd. Séances Acad. Sci. 207, 1018 [1938].

2 R. Chauvin, Ann. Soc. ent. France 110, 133 [1941].

3 T. W. Goodwin u. S. SRisukh, Biochem. J. 47, 549 [1950].

4 T. W. Goodwin, Biochem. J. 47, 554 [1950].

5 T. W. Goodwin, Biol. Rev. Cambridge Phil. Soc. 27, 439 [1952].
}

sichtsvolle Darstellung der Pigmente und ihres $\mathrm{Zu}$ sammenspiels haben uns Goodwin ${ }^{5}$ und kürzlich Uvarov $^{6}$ gegeben.

Bei Untersuchungen über die Verbreitung der Ommochrome ${ }^{7,8,9}$ waren wir ebenfalls auf die roten Pigmente der Heuschrecken gestoßen. Die ältere

\footnotetext{
${ }^{6}$ B. Uvarov, „Grasshoppers and locusts“, Vol. I, p. 42 ff., Cambridge 1966.

7 A. Butenandt, E. Biekert u. B. Linzen, Hoppe-Seyler's Z. physiol. Chem. 313, 251 [1958].

8 A. Butenandt, E. Biekert, H. Kübler u. B. Linzen, HoppeSeyler's Z. physiol. Chem. 319, 238 [1960].

9 B. Linzen, Zool. Anz. Suppl. 22, 154 [1959].
} 
Literatur zeigt erhebliche Meinungsverschiedenheiten über die Natur dieser Pigmente, dergestalt, daß Chauvin 1, 2,10 sie zu den Anthocyaninen stellte, Becker ${ }^{11}$ zu den Ommochromen, während GoodwIN $^{3,5}$ auf Grund seines Vergleiches verschiedener Augenpigmente die Unterscheidung der OmmochromUnterklassen Ommine und Ommatine aufhob und den Namen Ommochrome durch die neue Bezeichnung Insectorubin ersetzte. Wir legen jetzt Ergebnisse eigener Studien vor, die die Existenz mehrerer verwandter Pigmente, ihre Zugehörigkeit zu den Ommochromen und ihre Unterschiede von den bisher bekannten Ommochromen zeigen *.

\section{Beschreibung der Versuche}

\section{Papierchromatographie}

Sie erfolgte durchweg auf dem Papier No. $2043 \mathrm{~b}$ von Schleicher \& Schüll, aufsteigend in folgenden Systemen :

Collidin/Lutidin/Wasser $1: 1: 2$, org. Phase CLW 12 Collidin/0,5 M prim. Kaliumphosphat $2: 3$, org. Phase

Butanol/Eisessig/Wasser $4: 1: 1$

CP 12

Propanol/Ameisensäure/Wasser $1: 1: 4$

BEW

PAW

Chromatogramme, die im System CP gelaufen sind, zeigen unter UV-Licht eine Salzfront. Zur Ringpapierchromatographie wurde das System

Ameisensäure/Methanol/Wasser/konz. Salzsäure $50: 15: 20: 1$

AMWS

benutzt.

Zur Prüfung des Verhaltens der Farbstoffe bei Oxydation wurde mit einer Lösung von wenig Natriumnitrit in verd. Salzsäure betupft; zur Reduktion mit Ascorbinsäure oder Natriumdithionit in Wasser. Für die Reaktion mit Ninhydrin wurde mit einer 0,1-proz. Lösung von Ninhydrin in Butanol/Collidin/Eisessig (66:30:4) besprüht und anschließend 5-10 Min. auf $105^{\circ}$ erhitzt (in Anlehnung an Levy und CHUNG ${ }^{13}$ ).

\section{Isolierung von Augenpigment aus Wanderheuschrecken}

Aus etwa 1000 Köpfen von Locusta migratoria, die in Methanol aufbewahrt worden waren, wurden die Augen herausgeschnitten, getrocknet und fein gemahlen. Sie wurden je mehrere Male mit Äther und Äthanol extrahiert, und anschließend der Farbstoff mit salzsaurem Methanol (2 $\mathrm{ml}$ konz. $\mathrm{HCl}$ auf $100 \mathrm{ml}$ ), in das $\mathrm{SO}_{2}$ eingeleitet worden war, erschöpfend extrahiert. Der Extrakt wurde im Rotationsverdampfer eingedampft,

10 R. Chauvin, Bull. Soc. Zool. France 69, 154 [1944].

11 E. Becker, Z. Vererbungslehre 80, 157 [1942].

* Teilergebnisse wurden vorgetragen auf dem 17. int. Kongreß f. angew. Chemie, München 1959. in wenig reiner Ameisensäure aufgenommen und im System AMWS ringchromatographiert. Die Zone der Hauptkomponente wurde ausgeschnitten, mit Ameisensäure nach Art der absteigenden Papierchromatographie eluiert, und das Eluat zur Trockene gebracht. Das Pigment wurde mehrmals durch Lösen in $0,1 \mathrm{M}$ sek. Natriumphosphatlösung (wenn nötig, unter Zugabe von einigen Tropfen $\mathrm{N} \mathrm{NaOH}$ ) und erneutem Ansäuern mit $6 \mathrm{~N} \mathrm{HCl}$ und Einleiten von $\mathrm{SO}_{2}$ umgefällt, bis beim Lösen kein weißlicher Rückstand zurückblieb. Der Farbstoff wurde nun über eine Säule $(3,5 \cdot 15 \mathrm{~cm})$ von Ecteola-Cellulose chromatographiert. Der Austauscher wurde wie früher beschrieben ${ }^{8}$ vorbehandelt, in $1 \mathrm{M}$ Pyridinacetat von $p_{\mathrm{H}} 7,0$ vorgequollen und nach dem Einschlämmen in die Säule mit 0,1 M Natriumphosphatlösung gewaschen, bis der Pyridingeruch aus dem Eluat verschwunden war. Nach dem Auftragen des Farbstoffes wurde ebenfalls zunächst mit Natriumphosphatlösung gewaschen und dann mit einem PyridinPuffer-Gradienten eluiert: Im Mischgefäß $250 \mathrm{ml} 3 \mathrm{M}$ Pyridinwasser, im Zulauf $3 \mathrm{M}$ Pyridinformiat von $p_{\mathrm{H}}$ 6,5. Die Farbstoffbande wurde zur Trockene gebracht, 10-15-mal umgefällt, schließlich zweimal mit verdünnter $\mathrm{HCl}$, zweimal mit Aceton gewaschen und über $\mathrm{P}_{2} \mathrm{O}_{5}$ und Paraffin bei $80^{\circ}$ i. Vak. getrocknet. Ausbeute aus 1000 Köpfen : rund $12 \mathrm{mg}$.

\section{Versuche zur Charakterisierung des Farbstoffes}

Die Elementaranalysen wurden vom Laboratorium Dr. A. Schoeller (Kronach) und freundlicherweise von Herrn Dr. H. Reimlinger (Brüssel) ausgeführt. Sie ergaben:

C 41,20 H 4,22 N 17,94 O 27,07, Rückst. 5,5\%.

C 43,96 H 3,81, Rückst. 5,1\%.

Die Probe auf Schwefel wurde in der üblichen Weise vorgenommen und war bei dem Farbstoff sowohl mit Nitroprussidnatrium, als auch mit Bleiacetat positiv. Die Umsetzung mit Ninhydrin wurde nach Moore und STEIN ${ }^{14}$ durchgeführt.

Zur alkalischen Hydrolyse wurde eine Probe des Farbstoffes in $4 \mathrm{ml} \mathrm{N} \mathrm{NaOH}$ gelöst und auf dem siedenden Wasserbad erhitzt. Nach $0,1,2$ und 4 Stdn. wurden Proben von $0,4 \mathrm{ml}$ entnommen, verdünnt und die Extinktion zwischen 370 und $450 \mathrm{~nm}$ gemessen:

$\begin{array}{lcccc}\mathrm{nm} & 0 & 1 & 2 & 4 \text { Stdn. } \\ 370 & 0,585 & 0,600 & 0,720 & 0,750 \\ 400 & 0,900 & 0,890 & 0,960 & 0,980 \\ 450 & 0,445 & 0,410 & 0,453 & 0,462\end{array}$

Der Extinktionsanstieg nach 2 Stdn. geht auf Wasserverlust durch ungenügenden Verschluß des Kölbchens zurück. Nach Abbruch der Hydrolyse wurde die Lösung zur Trockene gebracht, mit wenig Pyridin und wenig

12 A. Butenandt, U. Schiedt, E. Biekert u. P. Kornmann, Liebig's Ann. Chem. 586, 217 [1954].

13 A. L. Levy u. D. Chung, Analytic. Chem. 25, 396 [1953].

14 S. Moore u. W. H. Stein, J. biol. Chemistry 176, 367 [1948]. 
absolutem Äthanol extrahiert und Proben dieser Extrakte in BEW und CLW papierchromatographisch untersucht. Der Rückstand wurde in wenig Wasser gelöst, der Farbstoff durch Einleiten von $\mathrm{SO}_{2}$ gefällt und in den Systemen CLW und AMWS chromatographiert.

In einem anderen Versuch wurde die alkalische Reaktionslösung angesäuert und nach Abtrennung des ausgefallenen Farbstoffes der saure Überstand mit Butanol extrahiert; unter diesen Bedingungen gehen die Abbauprodukte der Ommatine und Ommine (Xanthurensäure und 2-Amino-3-hydroxy-acetophenon) in die Butanolphase ${ }^{15}$. Die Butanolphase wurde unter $\mathrm{N}_{2}$ i. Vak. eingedampft, der geringe Rückstand mit salzsaurem Methanol gelöst und in $\mathrm{BEW}$ papierchromatographisch untersucht.

Zum Säureabbau wurden 1,23 mg des Farbstoffes in $3 \mathrm{ml}$ Ameisensäure gelöst, $3 \mathrm{ml} 6 \mathrm{~N}$ Salzsäure zugegeben und unter ständigem Durchleiten von $\mathrm{SO}_{2}$ während $20 \mathrm{Stdn}$. am Rückflußkühler gekocht. Die Lösung wurde im Rotationsverdampfer zur Trockene gebracht, der Rückstand mit wenig dest. Wasser befeuchtet, erneut eingedampft und nun mit wenig $\mathrm{SO}_{2}$-haltigem, salzsaurem Methanol gelöst und in den Systemen CLW, CP, BEW und AMWS papierchromatographisch untersucht.

\section{Einbau radioaktiver Vorstufen}

Alle Injektionen radioaktiver Substanzen erfolgten unter $\mathrm{CO}_{2}$-Betäubung seitlich in das Abdomen der Versuchstiere.

1 Tryptophan ${ }^{14} \mathrm{C} . \quad 970 \mu \mathrm{g} \quad(4,73 \mu \mathrm{M}) \quad$ D,L-Tryptophan-(Carboxyl- $\left.{ }^{14} \mathrm{C}\right)(0,66 \mathrm{mC} / \mathrm{mM})$ wurden in $0,3 \mathrm{ml}$ Insekten - R i n g e r - Lösung $\quad(7,5 \mathrm{~g} \mathrm{NaCl}, \quad 0,35 \mathrm{~g}$ $\mathrm{KCl}, 0,21 \mathrm{~g} \mathrm{CaCl}_{2}$ im $l$ ) unter Erwärmen gelöst und je $0,015 \mathrm{ml}$ in 16 Larven von Locusta migratoria injiziert. Nachdem sich alle Tiere zur Imago gehäutet hatten, wurden sie getötet, die Augen herausgeschnitten, getrocknet, gemahlen und das Pulver zweimal mit Benzol und anschließend mit salzsaurem Methanol extrahiert. Der Extrakt wurde eingedampft und der Rückstand mit 0,1 M sek. Natriumphosphat extrahiert. Dieses wurde wiederum zur Trockene gebracht und mit trockenem Äthanol, in das HCl-Gas eingeleitet worden war, extrahiert. Dieser Extrakt wurde papierchromatographisch in CLW getrennt, und die Farbstoffzone sowie die beiden Nachbarzonen, die noch wenig Farbstoff enthielten, ausgeschnitten. Sie wurden mit Äther und Aceton je zweimal ausgewaschen, und der Farbstoff mit Ameisensäure eluiert. Diese Eluate wurden auf Plättchen aufgetragen und ihre Aktivität gemessen.

Der Rest der Tierkörper (getrocknet 1,8 g) wurde gemahlen und mit Benzol und Methanol jeweils so lange extrahiert, bis der Extrakt farblos ablief. Darauf wurde mit $\mathrm{SO}_{2}$-haltigem, salzsaurem Methanol ein tiefroter Extrakt erhalten. Dieser wurde mit ges. Bicarbonatlösung auf $p_{\mathrm{H}} 5,0$ gebracht, ein geringer Nieder.

15 A. Butenandt, U. Schiedt u. E. Biekert, Liebig's Ann. Chem. 586, 229 [1954]. schlag abzentrifugiert und der Überstand mit dem fünffachen Volumen an Aceton versetzt. Der entstandene Niederschlag wurde abzentrifugiert, mit $\mathrm{SO}_{2}$-haltigem, salzsaurem Methanol digeriert und der weißliche Rückstand (Aceton-bisulfit-Verbindung) erneut abzentrifugiert. Der Überstand wurde papierchromatographisch im System PAW aufgetrennt. Man erhielt zwei rote Banden, die unter Schwefeldioxyd-Atmosphäre mit Ameisensäure eluiert und auf Zählplättchen aufgetragen wurden. Die Radioaktivität zeigte folgende Verteilung:

Untere Nachbarzone

Langsam wandernder Farbstoff

Zwischenzone (enthielt etwas Farbstoff) 127

Schnell wandernder Farbstoff

Obere Nachbarzone (enthielt noch Farbstoff) 115

Darauf folgende Zone

Ein Teil der Eluate wurde nochmals papierchromatographisch gereinigt und zeigte dann die in Abb. 5 wiedergegebene Verteilung der Radioaktivität.

2. 3-Hydroxy-kynurenin. Larven des letzten Stadiums von Gryllus bimaculatus wurden je ca. $3,9 \mu \mathrm{C}$ 3-Hydroxy-kynurenin- ${ }^{3} \mathrm{H}^{16}$ injiziert, und die Tiere nach

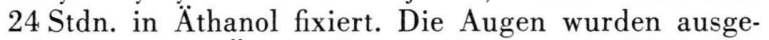
schnitten, unter Äther mit wenig Seesand zerrieben und dann analog der oben beschriebenen Isolierung des Ommidins verfahren. Die Säulenchromatographie wurde mit einer $0,5 \cdot 9 \mathrm{~cm}$ messenden Säule von DEAE-Sephadex und $25 \mathrm{ml} 3 \mathrm{M}$ Pyridinwasser im Gradienten-Mischgefäß sowie $50 \mathrm{ml}$ Pyridinformiat im Vorratsgefäß durchgeführt. Der Farbstoff wurde isoliert aufgefangen, i. Vak. zur Trockene gebracht und einmal umgefällt. Er wurde dann auf ein Ringchromatogramm aufgetragen, das nacheinander in den Systemen BEW, AMWS, dest. Wasser und wieder BEW entwickelt wurde. Zwischen den Läufen ließ man das Chromatogramm bei Zimmertemperatur trocknen. Das Chromatogramm wurde in ringförmige Zonen zerschnitten, der Farbstoff mit Ameisensäure eluiert, sein Spektrum gemessen und die Lösung erneut auf Filterpapierstreifen aufgetragen. Zur Messung der Aktivität im Flüssigkeits-Scintillationszähler wurden die Streifen im Sauerstoff-gefüllten Kolben verbrannt, und das Verbrennungswasser direkt mit dem Scintillationsgemisch (7 g PPO, 0,3 g Dimethyl-POPOP, $100 \mathrm{~g}$ Naphthalin, Dioxan ad $1 l$ ) aufgenommen.

3. Leucin ${ }^{14} C$. In dieser Aufarbeitung sollte die Wirksamkeit der Reinigung des Ommidins von möglicherweise radioaktiven Begleitstoffen geprüft werden. 18 Imagines von Gryllus bimaculatus wurden je $0,87 \mu \mathrm{C}$ D,L-Leucin- $\left(\right.$ Carboxyl- $\left.{ }^{14} \mathrm{C}\right) \quad(2,9 \mathrm{mC} / \mathrm{mM})$ injiziert, und nach 24 Stdn. der Augenfarbstoff wie vorstehend isoliert. Die Zonen des letzten Papierchromatogrammes wurden eluiert und die Aktivität der eluierten Substanz auf Plättchen mit einem Gas-Durchflußzähler

16 I. Ishiguro u. B. Linzen, Hoppe-Seyler's Z. Physiol. Chem. 340, 286 [1965]. 
(Tracerlab) gemessen. Die Farbstoffzone war absolut frei von Radioaktivität, während in einer der Nachbarzonen noch sehr schwache Radioaktivität gefunden wurde.

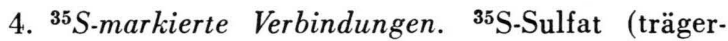
frei) und ${ }^{35} \mathrm{~S}$-markiertes Methionin (Ausgangsaktivität $12,9 \mathrm{mC} / \mathrm{mM}$ ) wurden Gruppen von je 15 Larven von Gryllus bimaculatus injiziert. Die Dosis betrug jeweils $10 \mu \mathrm{C}$ pro Tier; das Volumen der injizierten Lösung lag zwischen 11 und $25 \mu \mathrm{l}$. Die Tiere wurden nach $24 \mathrm{Stdn}$. fixiert und die Augen wie vorstehend für ${ }^{14} \mathrm{C}$ Leucin beschrieben aufgearbeitet. Bei den ersten Aufarbeitungen wurde in der Papierchromatographie statt des Systems AMWS das System CLW (aufsteigend) verwandt. Der Einbau von ${ }^{35} \mathrm{~S}$-Sulfat war entweder gleich Null oder äußerst niedrig (vgl. Tab. 1).

Die Verteilung der Radioaktivität im Ringchromatogramm nach Applikation von ${ }^{35} \mathrm{~S}-$ Methionin ist in Abb. 4 wiedergegeben.

\begin{tabular}{|c|ccccccccc|}
\hline Zone & Start & A & B & C & D & E & F & G & H \\
{$[\mathrm{IpM}]$} & 1 & 2 & 2 & 1 & 1 & 8 & 1 & 1 & 1 \\
\hline
\end{tabular}

Tab. 1. Verteilung der Radioaktivität im Papierchromatogramm des gereinigten Ommidins nach Injektion von Sulfat${ }^{35} \mathrm{~S}$ in Gryllus bimaculatus-Larven. Der Farbstoff befand sich in der Zone E. Werte sind IpM nach Abzug des Nullwertes.

\section{Vergleichende Untersuchung zum Vorkommen von Ommidin}

Die Augen der untersuchten Arten wurden entweder direkt auf Papierchromatogramme aufgequetscht, die in CLW oder CP entwickelt wurden, oder nach Trocknen und Zerreiben mit Äther und Methanol vorextrahiert und der Farbstoff mit $\mathrm{SO}_{2}$-haltigem, salzsaurem Methanol herausgelöst.

\section{Versuche zur Anreicherung der Epidermispigmente}

Die Aufarbeitung zum Nachweis des Einbaus von ${ }^{14}$ C-Tryptophan wurde bereits oben beschrieben. Für die Aufarbeitung größerer Materialmengen machten wir Vorversuche zur Trennung der Farbstoffe an Chromatographiesäulen. Dazu wurden jeweils 50-100 Köpfe von Schistocera gregaria, die in der rotgefärbten Schwärmphase standen, unter $\mathrm{SO}_{2}$-haltigem, salzsaurem Methanol zerrieben und nach Äbzentrifugieren noch mehrere Male mit dem gleichen Lösungsmittel extrahiert. Der Extrakt wurde i. Vak. zur Trockene gebracht, mit Aceton und Aceton/Methanol 1:1 extrahiert und das Farbstoffgemisch in $0,06 \mathrm{M}$ Phosphatpuffer von $p_{\mathrm{H}}$ 7,6-7,8, enthaltend $0,5 \%$ Natriumdithionit, gelöst. Die Lösung wurde auf Perlonpulver- oder Ecteolasäulen aufgetragen, und die Farbstoffe mit Phosphatund Pyridinacetat-Puffern verschiedenen $p_{\mathrm{H}}$-Wertes $\mathrm{zu}$ eluieren versucht. Die besten Ergebnisse erzielten wir auf Ecteola mit $2 \mathrm{M}$ Pyridinacetat von $p_{\mathrm{H}}$ 7,0. Die Chromatographie wurde in der Kälte durchgeführt, die Vorlagen mit Eis/Kochsalzmischung oder Aceton/Kohlensäureschnee gekühlt und die eingefrorenen Eluate lyophilisiert oder bei tiefer Temperatur im Rotationsverdampfer eingedampft. Die papierchromatographische Untersuchung erfolgte in den Systemen PAW und BEW.

\section{Ergebnisse}

\section{Papierchromatographische Analyse der braunroten Redoxfarbstoffe}

Die Augen der Wanderheuschrecken Locusta migratoria und Schistocerca gregaria sind nicht wie die der meisten anderen Insekten schwarzviolett oder dunkelbraun, sondern hellbraun gefärbt. Extrahiert man sie mit salzsaurem Methanol, so lassen sich papierchromatographisch 3 orangerote Pigmente nachweisen: Eine sehr kleine Menge Xanthommatin, eine ziegelrote Hauptkomponente und ein ebenfalls ziegelroter Begleitfarbstoff. Alle diese Farbstoffe können durch Nitrit in saurem Milieu zu einer gelben Form oxydiert und durch Ascorbinsäure oder Dithionit wieder zur roten Ausgangsform zurückgeführt werden. Während aber die Oxydation des Dihydro-xanthommatins schon durch Luftsauerstoff erfolgt, ist bei den ziegelroten Pigmenten die reduzierte Form beständig.

Im System CP liegt Xanthommatin auf der Salzfront, während die Hauptkomponente zwischen Startlinie und Salzfront mit $R_{f} 0,09$ wandert. In CLW wandert die Hauptkomponente mit $R_{f} \quad 0,2-0,3$, während sich Xanthommatin zersetzt und auch der Begleitfarbstoff einen breiten, verwaschenen Streifen bildet. In AMWS läuft der Hauptfarbstoff mit $R_{f} 0,55$, der Begleitfarbstoff mit $0,70-0,75$.

Zweifellos handelt es sich um Nativfarbstoffe, denn man erhält die gleichen Ergebnisse, wenn man Augen direkt auf die Startlinie der Chromatogramme aufquetscht. Der Anteil der Hauptkomponente wird auf $90-95 \%$ geschätzt.

Die beiden ziegelroten Farbstoffe wurden ausschließlich in den Augen nachgewiesen. In der Epidermis der Heuschrecken kommt jedoch ebenfalls rotes Pigment vor, das sich mit salzsaurem Methanol extrahieren läßt. Papierchromatographisch spaltet dieses Pigment in zwei Komponenten auf, von denen die in BEW und PAW schneller wandernde hellrot, die langsamer wandernde karminrot gefärbt ist. Auf dem Papier zeigen beide das für Ommochrome charakteristische Verhalten gegenüber Oxydationsund Reduktionsmitteln. Diese beiden Farbstoffe zersetzen sich so rasch, daß sie in Substanz bisher nicht gefaßt werden konnten (s. u.). Unsere Untersuchungen konzentrierten sich daher vorerst auf den ziegelroten Augenfarbstoff. 
Isolierung und Eigenschaften des Augenfarbstoffes

Dieser wurde aus einem Rohextrakt der Augen zunächst ringpapierchromatographisch von seinen Begleitpigmenten abgetrennt. Nach Elution mit Ameisensäure wurde er mehrmals umgefällt und über Ecteola rechromatographiert. Das eluierte Pigment wurde noch oftmals umgefällt; nach Auswaschen und Trocknen des Niederschlags erwies es sich als papierchromatographisch einheitlich und spektroskopisch sehr rein.

Der Farbstoff ist in Lipoidlösungsmitteln unlöslich; in Aethanol, Methanol und Wasser ist seine Löslichkeit gering, aber deutlich höher, als bei den bisher bekannten Ommochromen. Aus Methanolfixiertem Tiermaterial wird er jedoch nicht herausgelöst. Mäßig gut löslich ist er in salzsaurem Methanol oder Aethanol, in schwach alkalischen Pufferlösungen, gut löslich in Ameisensäure, Trifluoressigsäure (zu ca. 2-5\%), Dimethylsulfoxyd (zu ca. $2 \%$ ) und Natronlauge (über $10 \%$ ).

Der Farbstoff ist dialysierbar. Er enthält die Elemente $\mathrm{C}, \mathrm{H}, \mathrm{N}, \mathrm{O}$ und $\mathrm{S}$; mit den übrigen Ommochromen teilt er die sehr schwere Verbrennbarkeit, so daß brauchbare Elementaranalysen bisher nicht erhalten wurden. Möglicherweise enthält er eine Aminosäuregruppierung, da er eine positive Ninhydrin-Reaktion gibt. Mit $\mathrm{Ba}^{2 \oplus}$ bildet er ein schwerlösliches Salz.

Der Farbumschlag beim Ansäuern, den alle Ommochrome zeigen, ist hier besonders ausgeprägt und führt von hellgelb nach rot. Die UV-Spektren des Farbstoffes bei $p_{\mathrm{H}} 9$ (sek. Natriumphosphatlösung) und in $5 \mathrm{~N} \mathrm{HCl}$ sind in Abb. 1 dargestellt, ein Ausschnitt aus dem IR-Spektrum in Abb. 2. Einige Unterschiede von den bekannten Ommochromen lassen sich daraus ablesen. So fehlt im IR-Spektrum die Bande bei $5,77 \mu$, die der Carboxylgruppe am Chinolinring der Ommatine zugeordnet wird ${ }^{17}$. Im UV-Spektrum der angesäuerten Lösung fehlt die Bande bei $370 \mathrm{~nm}$ („C-Bande“), die bei allen Phenoxazonen gefunden wird, die in 3.4-Stellung einen aromatischen oder heterocyklischen Ring ankondensiert enthalten ${ }^{18}$. Außerdem verändert sich die Lage der Hauptextinktionsbande nicht; aber es tritt eine Anzahl von neuen Banden bei 440, 480 bis $500 \mathrm{~nm}$ hinzu. In 1-N Natronlauge wird das Maximum nach $403 \mathrm{~nm}$ verschoben.

17 A. Butenandt, E. Biekert, N. Koga u. P. Traub, HoppeSeyler's Z. physiol. Chem. 321, 258 [1960].

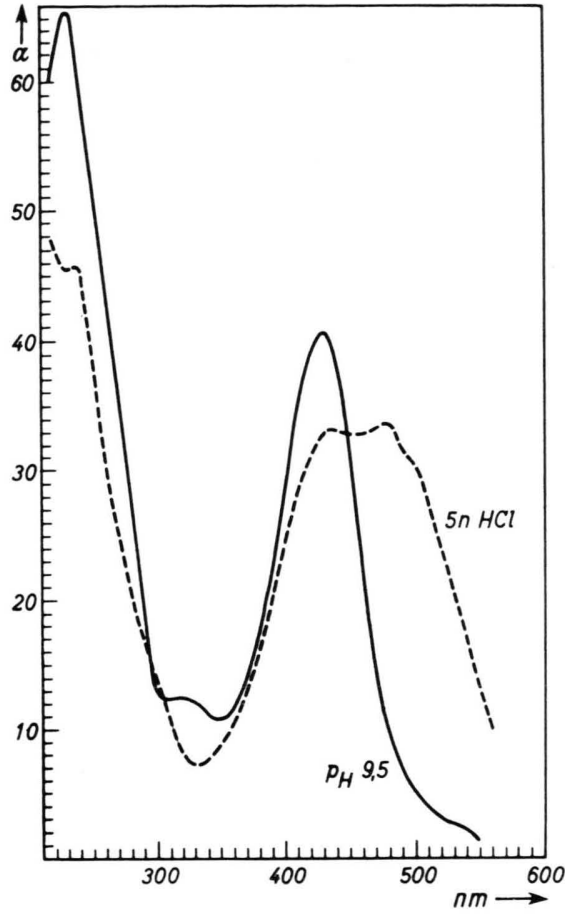

Abb. 1. UV-Spektren des Ommidins in 0,1 M sek. Natriumphosphatlösung (-) und in $5 \mathrm{~N} \mathrm{HCl}(---)$.

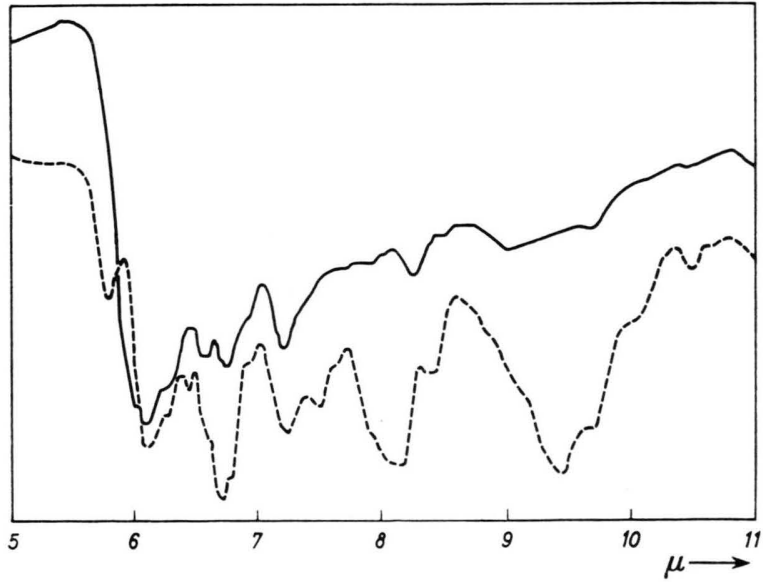

Abb. 2. IR-Spektrum des Ommidins (-) und zum Vergleich des Ommatins D $(---)$.

Obwohl der allgemeine Typus des Spektrums, besonders im schwach alkalischen Bereich, dem des Xanthommatins ähnelt, dürfte der chromophore Teil des Moleküls sich von den Ommatinen wie von den Omminen beträchtlich unterscheiden. - In konzentrierter Schwefelsäure zeigt der Farbstoff violette

\footnotetext{
18 A. Butenandt, U. Schiedt, E. Biekert u. R. J. T. Cromartie, Liebig's Ann. Chem. 590, 75 [1954].
} 
Halochromie mit einem Maximum bei $510 \mathrm{~nm}$ und mehreren Inflexionen unterhalb $320 \mathrm{~nm}$.

Das Kernresonanzspektrum weist folgende Signale auf: Bei 2,59 $\tau$ (Singulett), 2,82 $\tau$ (Singulett), $6,18 \tau$ (angedeutetes Triplett) und 6,66 $\tau$ (Multiplett), die zueinander die relativen Intensitäten 1:2:1:2 aufweisen. Der Bereich zwischen 3,9 und $6 \tau$ war (durch $\mathrm{H}_{2} \mathrm{O}$-Gehalt) nicht beobachtbar. Danach wären 3 aromatische Protonen im Molekül vorhanden, während die Signale bei 6,18 und 6,66 $\tau$ mit Vorbehalt einer Aminosäuregruppierung und einer $\mathrm{CH}_{2}$-Gruppe zugeordnet werden können.

Eine für uns überraschende Eigenschaft des Farbstoffes ist seine große Resistenz gegen Hydrolyse. Die Labilität besonders im alkalischen Milieu ist ja für Ommochrome charakteristisch. Als Extrem kann das Xanthommatin gelten, das schon bei $p_{\mathrm{H}} 8$ und Zimmertemperatur gespalten wird ${ }^{8}$; aber auch die Ommine werden beim Erwärmen in N Natronlauge in 2-3 Stdn. vollständig zersetzt und geben typische fluoreszierende Produkte ${ }^{19}$. Demgegenüber verändert sich der ziegelrote Augenfarbstoff der Heuschrecken auch in heißer Natronlauge nur langsam; nach $4 \mathrm{Stdn}$. wurde der größte Teil des Ausgangsmaterials unverändert zurückerhalten. Fluoreszierende Spaltprodukte konnten nicht nachgewiesen werden.

Auch die Stabilität gegen konzentrierte Säuren ist sehr groß, wenn man die Hydrolyse in reduzierendem Milieu durchführt; nach 20-stündigem Kochen in einem Gemisch von $6 \mathrm{~N} \mathrm{HCl}$ und konzentrierter Ameisensäure sind noch etwa $50 \%$ des Farbstoffes erhalten. Dagegen wird der Farbstoff in Anwesenheit von Sauerstoff durch Säure angegriffen; schon nach eintägigem Stehen in salzsaurem Methanol bemerkt man eine deutliche Zersetzung. Da der Farbstoff nativ offensichtlich in seiner reduzierten Form vorliegt und jedenfalls durch das reduzierende Extraktionsmilieu so erhalten wird, muß der Spaltung eine Oxydation vorausgehen. Tatsächlich lassen sich schon nach kurzer Einwirkung von Natriumnitrit gelbe, leicht wasserlösliche Spaltprodukte nachweisen. In keinem Falle konnte jedoch 3-Hydroxykynurenin als Reaktionsprodukt nachgewiesen werden.

Diese Eigenschaften - die große Stabilität des reduzierten und die Labilität des oxydierten Farb-

19 A. Butenandt, E. Biekert u. B. Linzen, Hoppe Seyler's Z. physiol. Chem. 312, 227 [1958]. stoffes - sind bei keinem der bekannten Ommochrome derart ausgeprägt, wohl aber bei einem Abbauprodukt der Ommine, dem „Farbstoff IV“ 20. Dieser enthält ebenfalls noch Schwefel, unterscheidet sich von dem Augenfarbstoff der Heuschrecken jedoch grundsätzlich in seinen UV-Spektren.

\section{Einbau radioaktiv markierter Vorstufen}

Die chemischen und physikalischen Eigenschaften des Farbstoffes, so sehr sie auf eine Verwandtschaft zu den Ommochromen hindeuten, sind dennoch für diese keineswegs beweisend. Ommochrome sind definitionsgemäß Tryptophanmetaboliten. Um ihnen den neuen Farbstoff zuzuordnen, mußte man seine Herkunft von dieser Aminosäure nachweisen. Dazu wurde Locusta-Larven Carboxyl $1{ }^{14} \mathrm{C}$-markiertes Tryptophan injiziert, und nach der Imaginalhäutung der Augenfarbstoff papierchromatographisch isoliert. Die Radioaktivität des Streifens fiel von der Startlinie gleichmäßig ab, zeigte jedoch einen Gipfel genau über der Farbstoffbande: Sie enthielt $51 \mathrm{IpM}$, die untere und obere Nachbarzone zusammen nur $16 \mathrm{IpM}$.

In weiteren Versuchen wurde Larven von Gryllus bimaculatus Tritium-markiertes 3-Hydroxy-kynurenin injiziert, und die Tiere nach jeweils 24 Stdn. fixiert. Der Farbstoff wurde isoliert und sorgfältig gereinigt. Da aus Untersuchungen an Omminen bekannt ist, daß diese Farbstoffe selbst nach intensiver Reinigung noch durch Protein verunreinigt sein können ${ }^{21}$, prüften wir unser Isolierungsverfahren durch eine Kontrollaufarbeitung von Tieren, die ${ }^{14} \mathrm{C}$-markiertes Leucin erhalten hatten. Dabei wurde der Farbstoff völlig frei von Radioaktivität gefunden. Nach Applikation von 3-Hydroxy-kynurenin dagegen befand sich nach dem letzten Reinigungsschritt ein großer Teil der Aktivität in der Farbstoffzone (Abb. 3). Daß auch noch außerhalb dieser Zone Aktivität gefunden wurde, dürfte auf den säurekatalysierten Abtausch von Tritium vom aromatischen Kern des Farbstoffes während der Entwicklung des Chromatogrammes in Ameisensäure zurückzuführen sein ${ }^{16}$.

Während der Befund, daß die Carboxylgruppe des Tryptophans im Farbstoff wiedererscheint, wegen des großen Zeitabstandes zwischen Applikation und Aufarbeitung und wegen der ungenügenden

\footnotetext{
20 A. Butenandt u. G. Neubert, Liebig's Ann. Chem. 618, 167 [1958].

21 B. Linzen, unveröffentlicht.
} 


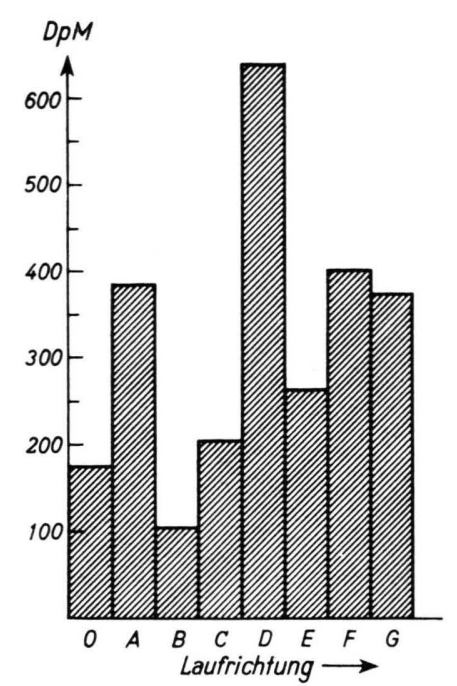

Abb. 3. Einbau von 3-Hydroxy-kynurenin ${ }^{3} \mathrm{H}$ in Ommidin aus Gryllus bimaculatus. Zone D enthält den Farbstoff (vgl. experimentellen Teil).

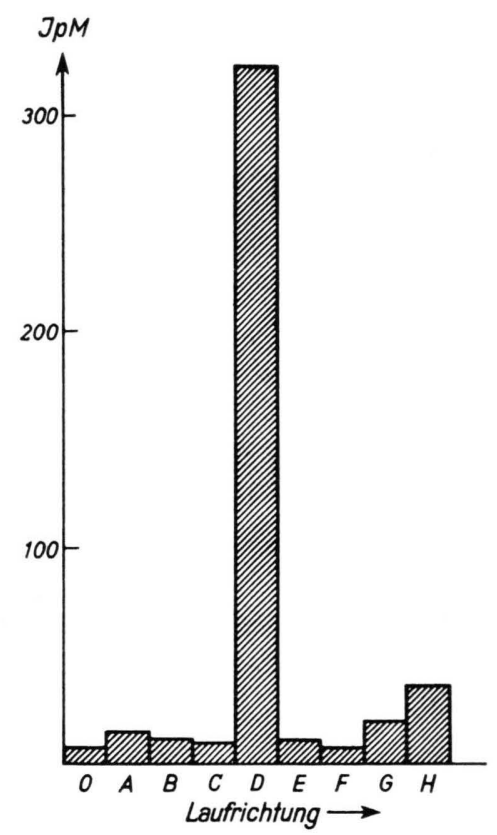

Abb. 4. Einbau von L-Methionin. ${ }^{35} \mathrm{~S}$ in Ommidin aus Gryllus bimaculatus. Zone D enthält den Farbstoff.

Reinigung noch anfechtbar ist, ist der Einbau von 3-Hydroxy-kynurenin sehr signifikant. 3-Hydroxykynurenin wird nach den bisherigen Kenntnissen im Insektenorganismus nur in Xanthurensäure, 3Hydroxy-anthranilsäure und in Ommochrome sowie in Konjugate dieser Verbindungen umgewandelt. Der Übergang von Radioaktivität aus dieser Verbindung in den Farbstoff zeigt also einen unmittel- baren Einbau der Substanz an und beweist, daß der Farbstoff ein Tryptophan-Metabolit ist wie das Xanthommatin, seine Konjugate und wie die $\mathrm{Om}$ mine.

Der ziegelrote Augenfarbstoff der Wanderheuschrecken und Grillen ist also einwandfrei den $\mathrm{Om}$ mochromen zuzuordnen. Er nimmt eine Mittelstellung zwischen den Ommatinen und Omminen ein, indem er mit den ersteren die hellere Farbe und die Dialysierbarkeit, mit den letzteren die größere Resistenz gegen Hydrolyse und den Schwefelgehalt teilt. Wir schlagen für ihn die Bezeichnung Ommidin vor und für den darunter sich verbergenden Begleitfarbstoff den Namen Kryptommidin.

Die Anwesenheit von Schwefel im Molekül, die zunächst nur durch die Lassaigne-Probe an teilweise gereinigten Präparaten gezeigt worden war, wurde in Versuchen zur Biosynthese von $\mathrm{Om}$ mochromen vollends bewiesen. Diese Versuche zeigen, daß markiertes Sulfat nicht oder nur in äußerst geringem Umfang in Ommidin eingebaut wird (vgl. Tab. 1). Dagegen wird ${ }^{35}$ S-markiertes Methionin sehr deutlich eingebaut (Abb.4), ganz parallel zu den Ergebnissen bei den Omminen von Gryllus $^{21}$. Auch diese Parallelität spricht dafür, daß Ommidin ein Ommochrom und möglicherweise den Omminen verwandt ist.

\section{Zur Verbreitung des Ommidins}

Das Vorkommen dieses Farbstoffes wurde bisher vor allem papierchromatographisch untersucht und ist in Tab. 2 dargestellt. Diese Liste ist zufällig entstanden und von beschränkter Aussagekraft. Man entnimmt daraus:

1. Die Isolierung des Ommidins aus Locusta oder aus Schistocerca ist kein Einzelbefund. Das Pigment kommt vielmehr in zwei Unterordnungen der Saltatoria vor.

2. Innerhalb der Insekten ist das Vorkommen des Ommidins wahrscheinlich auf die Orthopteren beschränkt. Jedenfalls wurde in 7 weiteren Ordnungen niemals Ommidin gefunden ${ }^{7,8,9}$.

3. Ein isoliertes Vorkommen des Ommidins ist das im Muskelansatzpigment von Limulus polyphemus. Es wurden aber nur Spuren des Pigments als Nebenfraktion bei einer Ommin-Aufarbeitung erhalten.

4. Xanthommatin ist ein regelmäßiger Begleiter des Ommidins in kleinen Mengen. Eine ähnliche 


\begin{tabular}{|c|c|c|c|}
\hline Art & unters. Organ & Farbstoff & Begleitfarbstoff \\
\hline $\begin{array}{l}\text { Schistocerca gregaria FoRsK. } \\
\text { Acanthacris ruficornis fulva SJöst. } \\
\text { Nomadacris septemfasciata SERV. } \\
\text { Ornithacris cyanea StoLL. } \\
\text { Tylotropidius speciosus WALK. } \\
\text { Locusta migratoria L. }\end{array}$ & $\begin{array}{c}\text { Augen } \\
\text { Integument } \\
\text { Augen } \\
\text { Augen } \\
\text { Körper } \\
\text { (Integument?) } \\
\text { Augen } \\
\text { Integument } \\
\text { Augen } \\
\text { Augen } \\
\text { Augen } \\
\text { Augen } \\
\text { Augen } \\
\text { Integument } \\
\text { Augen } \\
\text { Muskelansatz- } \\
\text { pigment }\end{array}$ & $\begin{array}{c}\text { Ommidin } \\
\text { Ommine } \\
\text { Ommidin } \\
\text { Ommidin } \\
\text { Ommidin } \\
\\
\text { Ommidin } \\
2 \text { rote Pigmente } \\
\text { Ommidin } \\
\text { Ommidin } \\
\text { Ommidin } \\
\text { Ommidin } \\
\text { Ommidin } \\
2 \text { rote Pigmente } \\
\text { Ommidin } \\
\text { Ommin }\end{array}$ & $\begin{array}{c}\text { Ommine } 21,22 \\
\text { Xanthommatin } \\
\text { Xanthommatin ? } \\
\text { Xanthommatin } \\
\text { Kryptommidin } \\
\text { Xanthommatin } \\
\text { Kryptommidin } \\
\text { Xanthommatin } \\
? \\
\text { Xanthommatin? } \\
? \\
\text { Xanthommatin } \\
\text { Kryptommidin } \\
\text { Xanthommatin } \\
\text { Xanthommatin } \\
? \\
\text { Ommidin }\end{array}$ \\
\hline
\end{tabular}

Tab. 2. Liste der Arten, in denen Ommidin nachgewiesen wurde. Einschränkungen: Die Identifizierung des Xanthommatins ist in keinem Falle gesichert; sie beruht lediglich auf der Beobachtung des gelbbraunen Redoxfarbstoffes auf der Salzfront von I'apierchromatogrammen im System CP. Auf Kryptommidin wurde z. Z. der vergleichenden Untersuchung noch nicht geachtet.

Rolle spielt es auch im Falle des Ommins. Bei Gryllus ist Ommidin mit Ommin vergesellschaftet ${ }^{22}$.

5. Ommidin tritt bei den Saltatoria fast ausschließlich als Augenfarbstoff auf. Erstmalig bei Romalea microptera wurde es - wiederum in Gesellschaft von Kryptommidin - in großer Menge in den Körpern gefunden. Das Integument wurde allerdings nur bei Locusta, Schistocerca und Gryllus genauer untersucht; auch bei einer genauen Analyse des Integuments von Carausius morosus ist Ommidin aber nicht gefunden worden ${ }^{23}$. Über die Verbreitung der beiden Hautpigmente kann man noch keine Angaben machen.

Bei Schistocerca ist seit einigen Jahren ein AlbinoStamm bekannt. Die Untersuchung des Augenpigmentes ergab, daß dessen Menge gleich groß wie beim Wildstamm ist. Bei beiden Stämmen wurde Ommidin gefunden; das Vorkommen sehr kleiner Mengen von Kryptommidin und Xanthommatin wird durch Papierchromatogramme nahegelegt. Die Ommochrome in den Augen beider Stämme unterscheiden sich also weder qualitativ noch quantitativ *.

22 S. Fuzeau-Braesch, C. R. hebd. Séances Acad. Sci. Paris 245, 2401 [1957].

23 J. H. Dustmann, Z. vergleich. Physiol. 49, 28 [1964].

* Anm. b. d. Korr.: Inzwischen wurde gezeigt (A. Bouthier, C. R. hebd. Séances Acad. Sci. 262, 1480 [1966]), daß

\section{Die roten Epidermisfarbstoffe von Locusta und Schistocerca}

Diese Farbstoffe konnten bisher nicht näher charakterisiert werden, da sie ganz ungewöhnlich instabil sind. In vivo sind sie in Granula der Epidermiszellen lokalisiert ${ }^{10},{ }^{24}$; sowie sie durch die Extraktion mit salzsaurem Methanol von diesem Trägerprotein abgelöst werden, beginnen sie sich zu braunem, schwer löslichem Material zu zersetzen. Es ist dies Verhalten, das Goodwin und SRIsukH ${ }^{3}$ bewog, die Ommine, die ja ebenfalls schwer löslich sind, als Kunstprodukte infolge der Alterung von „Insectorubin“ anzusehen. Für das Studium dieser Pigmente ist möglichst frisch in Methanol konserviertes Material zu verwenden. Es gelang uns nur, durch Arbeiten im sauren, höchstens neutralen, und reduzierenden Milieu die beiden Pigmente etwas anzureichern. In alkalischer Lösung zersetzen sie sich sofort; daher werden sie bei der Papierchromatographie in den Systemen CLW und CP, die üblicherweise bei der Untersuchung der Ommochrome benutzt werden, nicht gefunden. Ihre papier-

auch bei Locusta migratoria keine qualitativen und nur geringfügige quantitative Unterschiede in der Augenpigmentierung zwischen dem Wild- und einem Albinostamm bestehen.

24 D. Bellamy, Biochem. J. 70, 580 [1958]. 
chromatographische Trennung wurde bereits oben beschrieben.

Daß es sich auch bei diesen Farbstoffen um Ommochrome handelt, schließen wir einerseits aus ihrem Redox- und Löslichkeitsverhalten: Die Farbvertiefung bei Reduktion ist sehr charakteristisch, und die Löslichkeit in Fettlösungsmitteln, die sehr geringe Löslichkeit in Wasser, Methanol und Aethanol, das Verhalten als Säure und die Alkalilabilität weisen in die gleiche Richtung. Andererseits wurde in dem Versuch zur Markierung des Ommidins mit ${ }^{14} \mathrm{C}$-Tryptophan auch in den beiden Hautfarbstoffen Radioaktivität wiedergefunden. Die beiden Farbstoffe wurden papierchromatographisch getrennt, eluiert und rechromatographiert. Die Radioaktivität, die schon im ersten Chromatogramm deutliche Gipfel über den Farbstoffzonen zeigte (vgl. Zahlen im experimentellen Teil), war nach der 2. Chromatographie noch schärfer über diesen Zonen lokalisiert (Abb. 5).

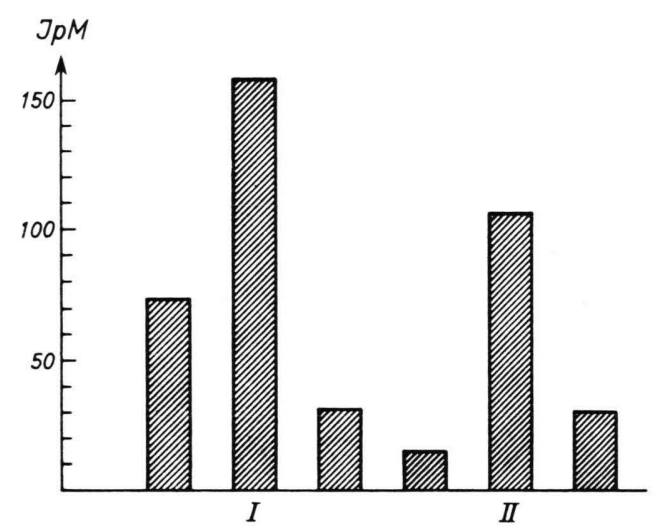

Abb. 5. Einbau von D,L-Tryptophan ${ }^{14} \mathrm{C}$ in die roten Epidermisfarbstoffe von Locusta migratoria. Farbstoff- sowie untere und obere Nachbarzonen nach Rechromatographie in PAW.

\section{Farbstoffe des Faeces}

Zur Zeit der Häutung und auch, wenn Heuschrecken hungern, ist der sonst olivbraune Kot der Tiere mehr oder weniger intensiv rot gefärbt ${ }^{5,25}$. Nach den Befunden älterer Arbeiten erfolgt die Ausscheidung des roten Pigmentes über die Mal. p igh i schen Gefäße. Nach papierchromatographischer Analyse besteht das rote Pigment aus mehreren Komponenten, von denen eine sich wie Xanthommatin verhält, während die beiden anderen in $\mathrm{BEW}$

25 R. Chauvin, C. R. Séances Soc. Biol. Filiales 130, 1194 [1939]. und PAW ähnlich wie die Hautfarbstoffe wandern. Die Farbstoffe wurden nicht näher untersucht, aber sind mit Sicherheit nicht mit den bekannten Xanthommatin-Derivaten im Exkretpigment anderer Insektenarten identisch ${ }^{8}$. Es sei darauf hingewiesen, daß rotes Pigment in sehr vielen Fällen bei der Puppenhäutung holometaboler Insekten gebildet wird, und daß die analoge Bildung von Pigment bei jungen Larven von Cerura vinula auch durch Nahrungsentzug experimentell ausgelöst werden kann ${ }^{26}$. Alle diese Pigmente, soweit sie untersucht worden sind, gehören zu den Ommochromen.

\section{Diskussion}

Die Farbstoffklasse der Ommochrome läßt sich seit einigen Jahren, nach der Reindarstellung und Strukturermittlung dreier Ommatine und nach der genaueren Beschreibung der Ommine, klarer überschauen ${ }^{27}$. Sie bot vorher ein Bild ähnlich den Melaninen - mit denen die Ommochrome im übrigen oft verwechselt wurden: Zwar konnte man Untergruppen beschreiben, die nach bestimmten Kriterien zu unterscheiden waren; aber die Grenzen schienen fließend zu sein, und z. B. die Ommine höhere Polymerisationsprodukte der gleichen Vorstufe, als die Ommatine. Wir wissen heute, daß die Ommine sich von den Ommatinen durch die Inkorporation eines weiteren Bausteines unterscheiden, der nicht aus dem Tryptophan-Stoffwechsel, sondern aus dem Methionin-Stoffwechsel stammt ${ }^{21}$.

$\mathrm{Zu}$ diesen nunmehr klar zu trennenden Gruppen treten jetzt das Ommidin und sein Begleiter, das Kryptommidin, hinzu. Auch das Ommidin leitet sich vom Tryptophan und vom Methionin ab, aber es läßt sich chemisch und physikalisch eindeutig gegen die Ommine abgrenzen. Wesentlich problematischer ist die Einordnung der beiden Hautfarbstoffe. Obwohl ihre Zugehörigkeit zu den Ommochromen ziemlich sicher erscheint, ist es bisher nicht möglich, irgendwelche Beziehungen zu den schon bekannten Ommochromen aufzustellen. Dies wäre besonders deshalb erwünscht, weil diese Farbstoffe eine wichtige Rolle bei der unterschiedlichen Färbung von Wander- und Solitärphase der Wanderheuschrecken spielen. Immerhin erscheint es zweckmäßig, die älteren Bezeichnungen „Acridioxanthin“ und „In-

\footnotetext{
${ }_{26}$ D. Bückmann, J. Ins. Physiol. 3, 159 [1959].

27 A. Butenandt, Naturwissenschaften 46, 461 [1959].
} 
sectorubin“ aufzugeben, zumal der erste Begriff nicht die in vivo rote Farbe der Pigmente trifft und beide Begriffe verallgemeinernd gemeint und angewendet wurden ${ }^{5}$.

Wie bei den meisten Farbstoffklassen der Tiere, kommen auch bei den Ommochromen fast immer mehrere Vertreter gleichzeitig in einer Tierart vor. Bei Locusta sind es 5 Ommochrome, bei Gryllus mindestens 7, wie wir in einer anderen Arbeit zeigen werden. Dies wirft die Frage auf, ob nicht die Entstehung mehrerer Ommochrome nebeneinander zufällig, anstatt funktionell zu verstehen wäre. Mehrere Befunde sprechen für die zufällig gemeinsame Bildung, sofern die Ommochrome strukturell sehr nahe verwandt sind:

1. Die parallel verlaufende Synthese von Rhodommatin und Ommatin D in der Metamorphose der Gabelschwanzraupe ${ }^{28}$.

2. Das stets gemeinsame Auftreten von Ommidin und Kryptommidin in den Augen der Heuschrecken.

3. Die Tatsache, daß vielfach kleine Mengen von Xanthommatin in Ommin-Gemischen gefunden werden. Das Auftreten kleiner Xanthommatin-Mengen ist überall dort wahrscheinlich, wo 3-Hydroxykynurenin oxydativ umgewandelt wird. - Die Synthese verschiedener Ommochrom-Typen - Ommine einerseits, Ommidine oder substituierte Ommatine andererseits - scheint dagegen eine spezifische Leistung bestimmter Tiergruppen oder - innerhalb einer Art - bestimmter Organe zu sein.

Die vorliegenden Versuche über die Biosynthese der Ommidine lassen noch keinen Schluß zu, auf welcher Stufe der Einbau des Schwefels erfolgt. Als Sulfat wie im Ommatin D liegt er sicher nicht vor. Der minimale Einbau von ${ }^{35} \mathrm{SO}_{4}{ }^{2 \ominus}$ kann jedenfalls

28 B. Linzen u. D. Bückmann, Z. Naturforschg. 16 b, 6 [1960]. zwanglos mit der Tätigkeit der Darmflora erklärt werden. Der Einbau von Methionin ist zweifellos signifikant, obwohl auch hier der Einbau, bezogen auf die applizierte Dosis, sehr niedrig ist. Das ist aber von vornherein zu erwarten: Nimmt man die Menge des Ommidins in einer Imago von Gryllus z. B. mit $10 \mu \mathrm{g}$ an, so dürfte die Syntheseleistung einer halberwachsenen Larve innerhalb von 24 Stdn. in der Größenordnung von $0,3 \mu \mathrm{g}$ liegen, eine Menge, die neben dem lebhaften Proteinstoffwechsel des wachsenden Tieres kaum ins Gewicht fällt.

Überhaupt ist der physiologische Zusammenhang der Pigmentbildung mit dem Methionin-Stoffwechsel bisher noch unklar. Da andere Insekten - z. B. die Schmeißfliege Calliphora ${ }^{29}$ - als Lichtschutzpigment im Blau-Grün-Bereich lediglich Xanthommatin, bzw. seine rote Dihydro-Form enthalten, dürfte der Schwefeleinbau kaum etwas mit einer spezifischen Lichtschutzfunktion zu tun haben. Er wird eher aus der Summe der physiologischen und ökologischen Bedingungen, die zur Selektion des Ommidins geführt haben, zu erklären sein.

Für ihr förderndes Interesse bin ich Herrn Prof. A. Butenandt und Herrn Prof. H. Dannenberg zu großem Dank verpflichtet. An der Durchführung der Arbeit waren beteiligt: Frl. G. RädEL, Frau I. KRONE, geb. KRöNNER, und vor allem Frl. R. PAGEL. Die IR-Spektren wurden von Frl. I. KöHLER, das KMR-Spektrum von Herrn Dr. J. Sonnenbichler und Frl. G. Schild angefertigt. ${ }^{14} \mathrm{C}$-markiertes Leucin wurde mir von Herrn Dr. W. ZILLIG zur Verfügung gestellt. Das Tiermaterial, soweit es nicht aus eigenen Zuchten gewonnen wurde, stammte vom Anti-Locust Research Centre (London), von Herrn Dr. W. Neupert und von Frau Dr. I. Laub-Drost. Die Arbeit wurde von der Deutschen Forschung s g emeinsch a f t finanziell wesentlich unterstützt. Allen sei an dieser Stelle herzlich gedankt.

29 A. Butenandt u. G. Neubert, Hoppe-Seyler's Z. physiol. Chem. 301, 109 [1955]. 